\title{
SET-TRANSITIVE PERMUTATION GROUPS
}

\author{
R. A. BEAUMONT AND R. P. PETERSON
}

1. Introduction. The concept of an $s$-ply transitive $(1 \leqslant s \leqslant n)$ permutation group on $n$ symbols is of considerable importance in the classical theory of finite permutation groups, which was in the height of its development in the period around the turn of the century. The obvious generalization to a permutation group which is $s$ set-transitive (i.e., a group which, for each pair of $s$-element unordered subsets $S, T$ of the given $n$ symbols, contains a permutation which carries $S$ into $T$ ) seems to have received little attention. A discussion (8, p. 257) of the symmetry of an arbitrary $n$-person game leads in a natural way to the notion of a set-transitive permutation group (i.e., a group which is $s$ set-transitive for all $s$ ) on the $n$ players of the game. In the preface to $(8)$, credit is given to $\mathrm{C}$. Chevalley for solving the problem of determining all set-transitive groups. Since, to our knowledge, nothing has appeared in the literature on this problem, we believe that a complete and relatively simple solution is of interest.

In $\$ 2$, the definitions are given, and the alternating and symmetric groups $A_{n}$ and $S_{n}$, along with the trivial cases for $n \leqslant 3$, are considered. The properties of $s$ set-transitive groups which are used for their enumeration are derived in $\S 3$, the principal results being contained in Theorems 5 and 6 , which state that these groups are transitive and primitive. In this connection, a recent paper by Bays (1), relating a concept of primitivity for ordered pairs to the degree of transitivity of a permutation group, is of interest. In $\$ 4$, a theorem on the distribution of prime numbers (2), is used to eliminate the possibility of settransitive groups for $n \geqslant 82$. Various special results in (5) and (7) are used to obtain Theorem 10, which states that set-transitive groups, other than $A_{n}$ and $S_{n}$, are possible only for $n=5,6$, and 9 . Finally, in $\S 5$, all of the settransitive groups of degree 5,6 , and 9 are determined. The results of this section are given in Theorem 11.

2. Definitions. We begin with a formal statement of the principal definitions and their immediate consequences. Since every permutation on $n$ symbols sends the complete set of $n$ symbols into itself, we exclude this trivial case from the following definition. Thus the case $n=1$ is excluded. Further, the identity group $I$ on $n$ symbols can clearly be omitted from consideration, so that in the sequel, by a group (\$), we will mean a permutation group (\$) $\neq I$ on the set $N$ of $n \geqslant 2$ symbols, $N=[1,2, \ldots, n]$.

Definition 1. A group (f) is $s$ set-transitive $(1 \leqslant s \leqslant n-1)$ if for every pair of subsets $S, T$ of $N=[1,2, \ldots, n]$, each containing $s$ elements, there exists a permutation in (\$) which carries $S$ into $T$.

Received October 9, 1952. 
It should be noticed that according to this definition, " 1 set-transitive" and "transitive" mean the same thing. We have the following immediate consequences of Definition 1:

(i) If the group $(\$)$ contains an $s$ set-transitive subgroup $\mathfrak{S}$, then (\$) is $s$ set-transitive.

(ii) If the group (S) is $k$-ply transitive, then (f) is $s$ set-transitive for all $s \leqslant k$.

(iii) If the group (S) contains permutations which carry the set $S=[1,2$, $\ldots, s]$ into any other set $T$ containing $s$ elements, then (\$) is $s$ set-transitive.

With $N=[1,2,3,4,5,6,7]$, the group $\$=\{(1234567),(235)(476)\}$, is an example of a group which is 2 set-transitive but not doubly transitive. (5) is not 3 or 4 set-transitive.

Definition 2. A group (5) is set-transitive if (5) is $s$ set-transitive for all $s(1 \leqslant s \leqslant n-1)$.

Since the alternating group $A_{n}(n \geqslant 3)$ is $(n-2)$-ply transitive and since $A_{2}$ is intransitive, we have

Theorem 1. The alternating group $A_{n}$ is set-transitive except for $n=2$. The symmetric group $S_{n}$ is set-transitive.

Proof. For $n \geqslant 3$, we have by (ii) that $A_{n}$ is $s$ set-transitive for all $s \leqslant n-2$. Since, in particular, $A_{n}$ is transitive, $A_{n}$ contains a permutation which sends $n$ into any other symbol $j$. Therefore $A_{n}$ contains a permutation which sends $S=[1,2, \ldots, n-1]$ into any other $n-1$ element set $T$. By (iii), $A_{n}$ is $n-1$ set-transitive, and hence set-transitive. Since $S_{n}$ is $n$-ply transitive for all $n, S_{n}$ is set-transitive.

It follows from the theorem that, in the determination of all set-transitive groups, we need only consider those groups $(5) \neq I$ which do not contain the alternating group. For $n=2$, there are none. For $n=3$, the only such groups are the three cyclic groups $\{(12)\},\{(13)\}$, and $\{(23)\}$ which are not 1 or 2 set-transitive. Having disposed of these trivial cases, we will henceforth assume that $n \geqslant 4$.

3. Properties of $s$ set-transitive groups. In this section we derive properties of $s$ set-transitive groups which are needed for their enumeration. The principal result is that an $s$ set-transitive group is primitive if $s>1$.

Theorem 2. A group (\$, which is the conjugate in $S_{n}$ of an set-transitive group $\mathfrak{S}$, is set-transitive.

Proof. Let $\sigma$ be a permutation in $S_{n}$ such that $(S)=\sigma \mathfrak{S} \sigma^{-1}$. Let $S=[1$, $2, \ldots, s]$, and $J=\left[j_{1}, j_{2}, \ldots j_{s}\right]$ be an arbitrary $s$-element subset. Define the set $I=\left[i_{1}, i_{2}, \ldots, i_{s}\right]$ by $\sigma^{-1} S=I$, and the set $K=\left[k_{1}, k_{2}, \ldots, k_{s}\right]$ by $\sigma^{-1} J=K$. Since $\mathfrak{S}$ is $s$ set-transitive, there exists a permutation $\tau \in \mathfrak{S}$ such that $\tau I=K$. Then

$$
\sigma \tau \sigma^{-1} \in \text { \$, } \quad \sigma \tau \sigma^{-1} S=\sigma \tau I=\sigma K=J .
$$


THEOREM 3. If the group \&f is s set-transitive, then \&f is $n-s$ set-transitive.

Proof. Let $S=[1,2, \ldots, n-s], C(S)=[n-s+1, n-s+2, \ldots, n]$. Let $J=\left[j_{1}, j_{2}, \ldots, j_{n-s}\right]$ be an arbitrary $(n-s)$-element subset, and $C(J)=\left[i_{1}, i_{2}, \ldots, i_{s}\right]$ be the complement of $J$ in $N$. There exists a permutation $\sigma \in \mathbb{B}$ such that $\sigma C(S)=C(J)$, so that $\sigma(S)=J$.

THEOREM 4. If the group \&5 is s set-transitive, then the order of \&5 is $m\left(\begin{array}{l}n \\ s\end{array}\right)$, where $m$ is the order of the subgroup $B_{1}$ of \&5 consisting of those permutations which carry the subset $S=[1,2, \ldots, s]$ into itself.

Proof. It is clear that the subset $\mathbb{S}_{1}$ of $(B)$ consisting of those permutations which carry $S=[1,2, \ldots, s]$ into itself is a subgroup. Since there are $t=\left(\begin{array}{l}n \\ s\end{array}\right)$ distinct $s$-element subsets of $N$, denote them by $I_{1}=S, I_{2}, \ldots, I_{t}$, and denote by $\sigma_{1}, \sigma_{2}, \ldots \sigma_{t}$ a set of permutations in \& such that $\sigma_{k} I_{1}=I_{k}$, for $k=1,2, \ldots, t$. Then $\sigma_{1}, \sigma_{2}, \ldots, \sigma_{t}$ form a complete set of representatives of (B) modulo $\mathbb{S}_{1}$, so that the order of $B$ is $m t$, where $m$ is the order of $\mathbb{S}_{1}$, and $t=\left(\begin{array}{l}n \\ s\end{array}\right)$.

COROLlary. If the group (5) is set-transitive, then the order of (5) is divisible by the least common multiple of the binomial coefficients

$$
\left(\begin{array}{l}
n \\
1
\end{array}\right),\left(\begin{array}{l}
n \\
2
\end{array}\right), \ldots,\left(\begin{array}{c}
n \\
n-1
\end{array}\right) \text {. }
$$

The 2 set-transitive group $B=\{(1234567),(235)(476)\}$ of degree 7 has order 21 . Since $\left(\begin{array}{l}7 \\ 2\end{array}\right)=21$, \& has minimum order for a 2 set-transitive group of this degree. Since

$$
\left(\begin{array}{l}
7 \\
3
\end{array}\right)=\left(\begin{array}{l}
7 \\
4
\end{array}\right)=35
$$

(5) cannot be 3 or 4 set-transitive.

THEOREM 5. If the group (S) is $s$ set-transitive for at least one $s$, then is is transitive.

Proof. Assume that $\mathbb{B}$ is intransitive and let $L \subset N$ be the smallest transitivity set of $\$ 5$. Then $L$ has $l$ elements where $l \leqslant \frac{1}{2} n$, and we may assume by Theorem 3 that $l \leqslant s$. Since $l \leqslant s \leqslant n-1$, there exists an $s$-element subset $S$ of $N$ such that $L \subseteq S$ and such that $C(S)$, the complement of $S$ in $N$, contains an element not in $L$. By removing an element of $L$ from $S$ and replacing it by an element from $C(S)$, we can construct an $s$-element subset $T$ such that $L \not \subset T$. Since $\mathbb{B}$ is $s$ set-transitive, there exists a permutation $\sigma \in \mathbb{B}$ such that $\sigma S=T$. Since $L$ is a transitivity set of $(\mathfrak{G}, \sigma L=L$, and since $L \subseteq S$, 
$\sigma L=L \subseteq \sigma S=T$. But this contradicts the choice of $T$, proving that (S) must be transitive.

THEOREM 6. If the group (s) is set-transitive for at least one $s>1$, then (5) is primitive.

Proof. By Theorem 3, we may suppose that $s \leqslant \frac{1}{2} n$. (5) is transitive by Theorem 5, and assume that $(5)$ is imprimitive. Then the set $N=[1,2, \ldots, n]$ can be partitioned into $r \geqslant 2$ subsets $N_{i}$, each containing $l \geqslant 2$ elements, such that every permutation in (5) carries each $N_{i}$ into some $N_{j}$.

If $s \leqslant l$, there is an $s$-element subset $S$ which is a subset of $N_{1}$. Since $s>1$, there is an $s$-element subset $T$ which contains elements from both $N_{1}$ and $N_{2}$. But since $(S)$ is $s$ set-transitive, there exists a permutation in $(S)$ which carries $S$ into $T$ and this contradicts the assumption that $(5)$ is imprimitive.

If $l<s$, then since $s \leqslant \frac{1}{2} n$, we have $l<\frac{1}{2} n$ and $r \geqslant 3$. Then there is an $s$-element subset $S$ which contains elements only from the sets of imprimitivity, $N_{1}, N_{2}, \ldots, N_{k}$ where $1<k \leqslant \frac{1}{2}(r+1)$, such that $S$ contains $N_{1}, N_{2}, \ldots$, $N_{k-1}$. Since $r \geqslant 3, r-k \geqslant \frac{1}{2}(r-1) \geqslant 1$, and the $r-k$ sets of imprimitivity, $N_{k+1}, \ldots, N_{r}$, are disjoint from those from which $S$ was constructed. Therefore there is an $s$-element subset $T$, constructed by replacing one of the elements of $N_{1}$ by an element from $N_{k+1}$, which contains elements from $k+1$ different sets of imprimitivity. Again, the existence of a permutation in (5) which carries $S$ into $T$ contradicts the assumption that $\$ 5$ is imprimitive.

In the next section, we use a classical result (3, p. 199) due to Jordan and Netto, which connects primitivity with the degree of transitivity of $\$$, to show that set-transitive groups are rare exceptions.

\section{Determination of the values of $n$ for which set-transitive groups may exist.}

THEOREM 7. If the group (5) is set-transitive $(s>1)$, and if there exists a prime $p$ such that $\frac{1}{2} n<p \leqslant n$ and such that $p$ divides $\left(\begin{array}{l}n \\ s\end{array}\right)$, then $\$$ is $(n-p+1)$ ply transitive.

Proof. Since the order of $(5)$ is $m\left(\begin{array}{l}n \\ s\end{array}\right)$ by Theorem 4 , if a prime $p$ divides $\left(\begin{array}{l}n \\ s\end{array}\right)$, (5) contains an element of order $p$. The elements of order $p$ in (5), when written as a product of cycles on disjoint letters, are products of cycles of length $p$. Since $p>\frac{1}{2} n$, the elements of order $p$ in $(S$ are cycles of length $p$. Such a cycle generates a cyclic subgroup $\mathfrak{S}$ of $\mathfrak{H}$ which is of degree $p$. Thus $\mathfrak{S}$ is primitive and leaves $n-p$ letters unchanged. Since $(5)$ is primitive by Theorem 6 , we obtain the conclusion of the theorem by employing the result mentioned above (3, p. 199) which states that if a primitive group (5) contains a primitive subgroup of degree $m$ which leaves the remaining $n-m$ letters unchanged, then (5) is $(n-m+1)$-ply transitive.

Corollary 1. If the group $(\$)$ is set-transitive $(s>1)$, and if there exists a prime $p$ such that $\max (s, n-s)<p \leqslant n$, then (5) is $(n-p+1)$-ply transitive. 
Proof. If $p>\max (s, n-s)$, then $p>\frac{1}{2} n$ and $p$ divides

$$
\left(\begin{array}{l}
n \\
s
\end{array}\right)=\frac{n !}{s !(n-s) !},
$$

so that the hypotheses of the theorem are satisfied.

In the determination of set-transitive groups, the critical value of $s$ is $s=\left[\frac{1}{2} n\right]$, where as usual this symbol denotes the greatest integer in $\frac{1}{2} n$.

CoROllary 2. If the group (5) is $\left[\frac{1}{2} n\right]$ set-transitive, and if there exists a prime $p$ such that $\frac{1}{2}(n+1)<p \leqslant n$, then (5) is $(n-p+1)$-ply transitive.

Proof. For $p>\frac{1}{2}(n+1) \geqslant\left[\frac{1}{2}(n+1)\right]=\max \left(\left[\frac{1}{2} n\right], n-\left[\frac{1}{2} n\right]\right)$, and the hypotheses of Corollary 1 are satisfied for $s=\left[\frac{1}{2} n\right]$.

We now make use of various known limits of transitivity to eliminate the possibility of the existence of $\left[\frac{1}{2} n\right]$ set-transitive, and therefore set-transitive, groups. The principal criterion is given in the following theorem.

THEOREM 8. If there exists a prime $p$ such that $\frac{1}{2}(n+1)<p<\frac{2}{3} n$, then a group \& on $n$ symbols, which does not contain the alternating group $A_{n}$, cannot be $\left[\frac{1}{2} n\right]$ set-transitive.

Proof. Assume that $\left(5\right.$ is $\left[\frac{1}{2} n\right]$ set-transitive. Then if a prime $p$ exists in the given range, (5) is $(n-p+1)$-ply transitive by Corollary 2 , Theorem 7 . But since $\frac{1}{3} n+1=n-\frac{2}{3} n+1<n-p+1$, and since $\frac{1}{3} n+1$ is an upper limit for the degree of transitivity (3, p. 152) for a group \& not containing $A_{n}$, we have a contradiction.

There are many refinements of Bertrand's postulate which states that a prime exists in the range between $x$ and $2 x$. One such result which is convenient for our purposes is due to Breusch (2). He shows that for $x \geqslant 48$, there always exists a prime between $x$ and $9 x / 8$. For $n \geqslant 82, x=\frac{1}{2}(n+14)$ $\geqslant 48$, and there exists a prime between $\frac{1}{2}(n+14)$ and $9(n+14) / 16$. Since $\frac{1}{2}(n+1)<\frac{1}{2}(n+14)$, and $9(n+14) / 16<\frac{2}{3} n$ for $n \geqslant 82$, there exists a prime in the range given in the hypothesis of Theorem 8. By examining a table of primes, we find a prime strictly between $\frac{1}{2}(n+1)$ and $\frac{2}{3} n$ for all $n \geqslant 26$, and we have

THEOREM 9. A group (5) on $n \geqslant 26$ symbols, which does not contain the alternating group $A_{n}$, cannot be $\left[\frac{1}{2} n\right]$ set-transitive, and therefore is not settransitive.

For $n<26$, a table of primes shows that a prime lies in the required range for $n=8,11,12$ and for all $n$ such that $17 \leqslant n \leqslant 24$. Since the cases for $n<4$ have been previously discussed, we have only the cases

$$
n=4,5,6,7,9,10,13,14,15,16,25
$$

to investigate. For these cases we first employ a better result on the limit of transitivity due to Miller (7, vol. III, p. 439) which states that if $n=k p+r$, 
where $p$ is a prime greater than the positive integer $k$ and where $r>k$, then a group (5) on $n$ symbols, not containing the alternating group $A_{n}$, cannot be more than $r$-ply transitive, unless $k=1$ and $r=2$. As an example, with $n=25$, we obtain from Corollary 2 , Theorem 7 with $p=17$, that if $\$$ is 12 set-transitive, then $(5)$ is 9 -ply transitive. But $25=1 \cdot 19+6$ with $k=1$, $p=19$, and $r=6$, so that \$f cannot be more than 6 -ply transitive. Therefore there are no groups on $n=25$ symbols, other than $A_{25}$ and $S_{25}$, which are $\left[\frac{1}{2} n\right]=12$ set-transitive. In this way, the cases $n=10,14,15,16$, and 25 are eliminated.

Miller has proved (7, vol. I, p. 200) that a transitive group of degree 13, which does not contain the alternating group $A_{13}$, is at most doubly transitive. By Corollary 2, Theorem 7 with $p=11$, we have that if $(5)$ is $\left[\frac{1}{2} n\right]=6$ settransitive, then \& is triply transitive. Similarly, for $n=7$, a transitive group not containing $A_{7}$ is at most doubly transitive $(5$, p. $186 ; \mathbf{6}$, p. $338 ; \mathbf{7}$, vol. I, pp. 1-9), while Corollary 2 , Theorem 7 , with $p=5$, gives that if $(5)$ is $\left[\frac{1}{2} n\right]=3$ set-transitive, then $(5)$ is triply transitive.

A 2 set-transitive group (\$) on $n=4$ symbols has an order divisible by $\left(\begin{array}{l}4 \\ 2\end{array}\right)=6$ by Theorem 4 . The only such transitive groups are $A_{4}$ and $S_{4}(6$, p. $338 ; 7$, vol. I, pp. 1-9). The cases $n=2$ and 3 were eliminated in $\S 1$.

We summarize the above results in the following theorem.

Theorem 10. A group (5) on $n$ symbols, which does not contain the alternating group $A_{n}$, cannot be $\left[\frac{1}{2} n\right]$ set-transitive, and therefore is not set-transitive, with the exceptions of $n=5,6$, and 9 .

5. Determination of the set-transitive groups for $n=5,6$, and 9 . In the following determination of set-transitive groups, we use the table of transitive groups on $n \leqslant 9$ symbols given by Cole in (6). Although this list has two omissions for $n=8$, it has been verified by Miller (7, vol. I, pp. 1-9, 12-14) and others for $n=5,6$, and 9 .

If a group $\$$ on 5 symbols is 2 set-transitive, then by Theorem 4 the order of (5) is divisible by $\left(\begin{array}{l}5 \\ 2\end{array}\right)=10$. Thus the only possibilities for a 2 set-transitive group, other than $A_{5}$ or $S_{5}$, are the transitive groups:

$$
\begin{aligned}
& G_{1}=\{(12345),(1325)\}, \\
& H_{1}=\{(12345),(12)(35)\},
\end{aligned}
$$

order 20 , order 10 ,

and their conjugate subgroups in $S_{5}$. Since $G_{1}$ is doubly transitive, it is 2 settransitive, and therefore 3 set-transitive by Theorem 3 . Since $G_{1}$ is transitive, it is 4 set-transitive by this same theorem. Thus $G_{1}$ and its conjugate subgroups in $S_{5}$ (Theorem 2) are set-transitive. If $H_{1}$ were 2 set-transitive, then the order of $H_{1}$ would be $10 \mathrm{~m}$, where $m$ is the order of the subgroup of $H_{1}$ which carries the set $[1,2]$ into itself. Thus $m=1$, and the identity is the only 
element of $H_{1}$ which carries [1,2] into itself. But $(12)(35) \in H_{1}$ carries $[1,2]$ into itself. Therefore $H_{1}$ is not 2 set-transitive.

By Theorem 4, if a group $\$ 5$ on 6 symbols is 3 set-transitive, then the order of $(5)$ is $m\left(\begin{array}{l}6 \\ 3\end{array}\right)=20 m$, where $m$ is the order of the subgroup of (5) which sends the set $[1,2,3]$ into itself. The only possibilities for a 3 set-transitive group, other than $A_{6}$ or $S_{6}$, are the transitive groups:

$$
\begin{aligned}
G_{2} & =\{(12345),(12)(35),(13465),(1325)\}, & & \text { order } 120, \\
H_{2} & =\{(12345),(12)(35),(13465)\}, & & \text { order } 60,
\end{aligned}
$$

and their conjugate subgroups in $S_{6}$. Since $G_{2}$ is triply transitive, it is both 3 and 2 set-transitive, and by Theorem $3, G_{2}$ is set-transitive, as are its conjugates in $\mathrm{S}_{6}$. If $\mathrm{H}_{2}$ were 3 set-transitive, then the order of the subgroup of $\mathrm{H}_{2}$ which carries the set $[1,2,3]$ into itself would be 3 . However, the permutations

$$
\text { (1), (13) (45), (123) (465), (23) (56) }
$$

in $H_{2}$ carry $[1,2,3]$ into itself. Therefore $H_{2}$ is not 3 set-transitive.

Again using Theorem 4, a 4 set-transitive group on 9 symbols has order $m\left(\begin{array}{l}9 \\ 4\end{array}\right)=126 m$. The only possibilities, other than $A_{9}$ and $S_{9}$, are the transitive groups:

$$
\begin{aligned}
G_{3} & =\{(1254673),(15)(29)(47)(68),(124)(765)\}, & & \text { order 1512, } \\
H_{3} & =\{(1254673),(15)(29)(47)(68)\}, & & \text { order 504, }
\end{aligned}
$$

and their conjugate subgroups in $S_{9}$. Since $H_{3}$ is triply transitive, $H_{3}$ is 1,2 , $3,6,7$ and 8 set-transitive by Theorem 3 . By this same theorem if $H_{3}$ is 4 settransitive, it is 5 set-transitive and consequently set-transitive. That a permutation can be found in $H_{3}$ which sends the set $[1,2,3,4]$ into each of the 126 four element subsets of $N=[1,2,3,4,5,6,7,8,9]$ has been checked directly by the authors. Therefore $H_{3}$ is set-transitive, and since $H_{3}$ is a subgroup of $G_{3}$, $G_{3}$ is also set-transitive. We summarize these results in the following theorem which, as was indicated at the beginning of this section, depends in part on the correctness of the list of transitive groups $(6, \mathrm{p} .338)$ for $n=5,6$, and 9 .

THEOREM 11. The only groups on $n$ symbols, other than the symmetric and alternating groups $S_{n}$ and $A_{n}$, which are $\left[\frac{1}{2} n\right]$ set-transitive, are the groups $G_{1}$, $G_{2}, H_{3}$, and $G_{3}$, and their conjugates, on 5, 6, 9, and 9 symbols, respectively. These four groups are set-transitive.

In the verification that the group $H_{3}$ is 4 set-transitive, an element of the form $\tau \sigma^{n}$, where $\tau$ is an element of order two or three in $H_{3}$ and $\sigma=(1254673)$ $\in H_{3}$, can be found which carries the set $[1,2,3,4]$ into each four element subset $[a, b, c, d]$. For example, with $\tau=(64)(72)(51)(39)$ and $n=4, \tau \sigma^{n}$ carries $[1,2,3,4]$ into $[2,3,5,9]$. In the same way, with $\tau=(756)(412)(839)$ and $n=0,1,4,5,6, \tau \sigma^{n}$ carries $[1,2,3,4]$ into $[1,2,4,9],[2,5,6,9],[1,6,7,9]$, $[2,3,7,9]$, and $[1,3,5,9]$, respectively. 
It may be of some interest to give an additional description of the four set-transitive groups $G_{1}, G_{2}, H_{3}$, and $G_{3}$. As an abstract group, $G_{1}$ is metacyclic with defining relations $R^{5}=S^{4}=1, S^{-1} R S=R^{2}$ (7, vol. III, p. 241). $G_{2}$ is isomorphic to the symmetric group $S_{5}$, and has the abstract defining relations $R^{5}=S^{4}=\left(R S^{2}\right)^{3}=\left(R^{3} S\right)^{2}=1$ (7, vol. III, p. 241). The group $H_{3}$ is the simple group $L F(2,8)$ consisting of all the linear fractional transformations

$$
x^{\prime}=\frac{a x+b}{c x+d}
$$

where $a, b, c, d$ are elements of $G F\left(2^{3}\right)$ such that $a d-b c \neq 0$. As an abstract group, $H_{3}=\{A, B\}$ has defining relations

$$
A^{7}=B^{2}=(A B)^{3}=\left(A^{3} B A^{5} B A^{3} B\right)^{2}=1
$$

(4, p. 174). Finally, $G_{3}$ is isomorphic to the group of automorphisms of $H_{3}$, and $G_{3}=\{A, B, C\}$, where $C$ satisfies the relations

$$
C^{3}=1, C A C^{-1}=A^{2}, C B C^{-1}=A B A^{4} B A^{4} B A .
$$

Thus $G_{3}$ has order 1512 and contains $H_{3}$ as a normal subgroup of index 3 . The generators $A, B$, and $C$ are given by $A=(1254673), B=(15)(29)(47)(68)$, and $C=(124)(765)$ in terms of the given generators of $H_{3}$ and $G_{3}$ as permutation groups on nine symbols.

\section{REFERENCES}

1. S. Bays, Sur la transitivité et la primitivité des groupes de substitutions, Comm. Math. Helvet, 22 (1949), 17-30.

2. R. Breusch, Zur Verallgemeinerung des Bertrandschen Postulates, das zwischen $x$ und $2 x$ stets Primzahlen liegen, Math. Zeitschrift, 34 (1932), 505-526.

3. W. Burnside, Theory of groups (Cambridge, 1897).

4. - - Note on the simple group of order 504, Math. Ann., 52 (1899), 174-176.

5. F. N. Cole, Note on the substitution groups of six, seven, and eight letters, Bull. New York Math. Soc., 2 (1893), 184-190.

6. - List of the substitution groups of nine letters, Quart. Jour. Math., 26 (1893), 372-388.

7. G. A. Miller, Collected works, vols. I and III (Urbana, 1935 and 1946).

8. J. von Neumann and O. Morgenstern, Theory of games and economic behavior (Princeton, 1947).

University of Washington 